Bulletin of Pharmaceutical Sciences
Assiut University
Website: http://bpsa.journals.ekb.eg/
e-mail: bullpharm@aun.edu.eg

\title{
IDENTIFICATION AND CHARACTERIZATION OF METALLO- $\beta$ - LACTAMASES PRODUCING PSEUDOMONAS AERUGINOSA CLINICAL ISOLATES IN AL-AZHER UNIVERSITY HOSPITAL, ASSIUT
}

\author{
Rehab Mousa ${ }^{1,4^{*}}$, Magdy F. El-Kady ${ }^{2}$, Asmaa A. A. Hussein ${ }^{3}$, Amr E. Ahmed ${ }^{4}$ and \\ Ahmed Osama El-Gendy ${ }^{5}$ \\ ${ }^{1}$ Pharmacist at Al-Azher University Hospital, Assiut, Egypt \\ ${ }^{2}$ Department of Poultry Diseases, Faculty of Veterinary Medicine, Beni-Suef University, Beni- \\ Suef, Egypt \\ ${ }^{3}$ Department of Zoonoses, Faculty of Veterinary Medicine, and Dean of Molecular Biology \\ Researches \& Studies Institute, Assiut University, Assiut, Egypt \\ ${ }^{4}$ Deparmtent of Biotechnology and Life Sciences, Faculty of Postgraduate Studies for \\ Advanced Sciences, Beni-Suef University, Beni-Suef, Egypt \\ ${ }^{5}$ Department of Microbiology and Immunology, Faculty of Pharmacy, Beni-Suef University, \\ Beni-Suef, Egypt
}

\begin{abstract}
Purpose: Study aimed to determine the frequency of nosocomial infections caused by $P$. aeruginosa and its distribution among different intensive care units (ICUs), to determine the antibiotic resistance pattern, and to determine the prevalence of Metallo- $\beta$-lactamase (M $\beta L)$ among isolates by phenotypic methods. Methods: A total of 74 P. aeruginosa isolates were identified from different clinical specimens in AL-Azher University Hospital. The antimicrobial susceptibility was tested by disk diffusion (Kirby-Bauer) method and phenotypic screening for $M \beta L s$ was performed using Combined Disk Test (CDT) and double-disk synergy test (DDST). Results: The frequency of $P$. aeruginosa isolates from clinical specimens was 18\% (74/412). The highest sensitivity was to imipenem 38 isolates (51.3\%). The antibiotic sensitivity was in descending manner to meropenem $(48.6 \%)>$ levofloxacin $(28.4 \%)>$ ciprofloxacin and ceftazidime (21.6\%), while the highest resistance rates were to carbenicillin 70 isolates $(94.6 \%)$ then gentamicin (70.3\%). Out of 74 P. aeruginosa isolates, 51/74 (69\%) strains were multidrug resistant (MDR), based on the CDT results $26 / 74$ (35.1\%) isolates and by DDST 23/74 (31.1\%) isolates were confirmed to be $M \beta L$ s producers. Conclusion: There is a growing risk for isolation of MDR P. aeruginosa among the MBL-producer isolates suggests the need for continuous assessment of antimicrobial susceptibility and surveillance of antibiotic prescription. In addition, infection control measures are needed to prevent further dissemination of these organisms.
\end{abstract}

\section{INTRODUCTION}

Infectious diseases are one of the leading causes of death worldwide due to the development of antimicrobial resistance ${ }^{1} . P$. aeruginosa is one of the most prevalent opportunistic human pathogen causing several clinical infections including wound infection, pneumonia, urinary tract infections, endocarditis, meningitis, brain abscess, and bacteremia $^{2 \& 3}$. The increasing inappropriate use of broad-spectrum antibiotics has increased the emergence of multidrug resistant $P$. aeruginosa (MDRPA) isolates which complicates the

Received in 11/10/2020 \& Accepted in 26/11/2020 
process of therapy and limits treatment options ${ }^{4}$. Multidrug resistant is defined as being resistant to at least 3 anti-pseudomonal antibiotic-groups including $\beta$-lactams, aminoglycosides, and fluoroquinolones 5 . The growing threat of antimicrobial resistance in $P$. aeruginosa relays on one hand in the extraordinary capacity of this microorganism for developing resistance to almost any available antibiotic by the selection of mutations in chromosomal genes, and on the other, in the increasing prevalence of transferable resistance determinants, particularly those encoding class B carbapenemases (or metallo- $\beta$-lactamases $(\mathrm{M} \beta \mathrm{L})^{6}$. Metallo- $\beta$-lactamase $\quad(\mathrm{M} \beta \mathrm{L})$ production is increasingly reported as a cause of high-level carbapenem resistance among $P$. aeruginosa, an important nosocomial pathogen that is notorious for multi-drug resistance ${ }^{7}$. Genes encoding M $\beta$ Ls are generally located within class 1 integrons together with those encoding aminoglycoside modifying enzymes, which therefore confer $\mathrm{MDR}^{8}$.

\section{PATIENTS AND METHODS}

In this study, seventy four $P$. aeruginosa isolates were obtained from clinical specimens submitted for bacteriological testing from hospitalized in-patients admitted to AL-Azher University Hospital, and Molecular Biology Research Unit, Assiut University, Egypt during the period between November 2015 to April 2017.

\section{Isolation and identification of $\boldsymbol{P}$. aeruginosa isolates}

These isolates were identified by standard laboratory methods including bacteriologic and biochemical methods such as; Gram staining, colony morphologies on MacConkey's agar, biochemical tests such as oxidase, catalase, Oxidative-fermentative test, growth on media such as Triple Sugar Iron Test (TSI), cetrimide agar and growth at $42^{\circ} \mathrm{C}$. These isolates were stored at $-70^{\circ} \mathrm{C}$ in trypticase soy broth containing $20 \%$ glycerol and sub cultured twice prior to testing.

\section{Antimicrobial susceptibility testing}

The susceptibility pattern of isolates to different antibiotics were examined using disk diffusion method (Kirby-Bauer) on MullerHinton agar plates according to Clinical and Laboratory Standards Institute (CLSI) ${ }^{9}$. The antimicrobial disks were included: imipenem (10 $\mu \mathrm{g})$, meropenem $(10 \mu \mathrm{g})$, ceftazidime (30 $\mu \mathrm{g})$, carbenicillin $(100 \mu \mathrm{g})$, amikacin $(30 \mu \mathrm{g})$, gentamicin $(10 \mu \mathrm{g})$, Ciprofloxacin $(5 \mu \mathrm{g})$, Levofloxacin $(5 \mu \mathrm{g})$. inoculated plates and incubated overnight. The zones of inhibition were measured and interpreted.

MIC Determination for $P$. aeruginosa isolates by Epsilometer test ( $E$ test)

Determination of MIC $P$. aeruginosa isolates by Imipenem (IP)-E test and Meropenem (MP)-E test (bioMérieux SA, Marcy l'Etoile, France). Results were interpreted using CLSI (2016) ${ }^{10}$ breakpoints. The breakpoints for imipenem and meropenem were as follows: susceptible $(\mathrm{S}) \leq 4 \mu \mathrm{g} / \mathrm{ml}$ and resistant $(\mathrm{R}) \geq 16 \mu \mathrm{g} / \mathrm{ml}$.

MBL screening and confirmation by phenotypic methods

Combination disk diffusion test (CDDT) was used for phenotypic detection of MBLs producing $P$. aeruginosa strains. In brief a 0.5 M EDTA solution was prepared and then was added to $10 \mu \mathrm{g}$ imipenem and meropenem disks to obtain a concentration of $750 \mu \mathrm{g}$. The IPM and IPM -EDTA disks were placed on the plate which were inoculated with $P$. aeruginosa. The inhibition zones of these disks with and without EDTA solution were compared after $16-18 \mathrm{hrs}$ of incubation in air at $35^{\circ} \mathrm{C}$. An increase of $\geq 7$ in the zone diameter for IPM in the presence of EDTA was considered as positive result.

Double-disk synergy test (DDST), in brief a $10 \mu \mathrm{l}$ of $0.5 \mathrm{M}(750 \mu \mathrm{g})$ EDTA solution was added to filter paper blank disc, was placed on the surface of the agar plate. IPM disc $(10 \mu \mathrm{g})$ or MEM disc $(10 \mu \mathrm{g})$ was placed $13 \mathrm{~mm}$ center to center from the EDTA disc. The plates were incubated for $16-18 \mathrm{hrs}$ at $35^{\circ} \mathrm{C}$. Enhancement of the inhibition zone in the area between the imipenem (or meropenem) disc and the EDTA disk in comparison with the inhibition zone on the far side of the drug was interpreted as positive for $\mathrm{M} \beta \mathrm{L}$ production ${ }^{11}$. 


\section{RESULTS AND DISCUSSION}

\section{Results}

Isolation and Identification of pathogens were done on specimens according to standard procedures. 412 isolates from different clinical samples were collected. Out of 412 isolates, Enterobacteriaceae $s p$. were the most common isolates detected, they were $226(54.85 \%)$. The incidence of other microorganisms was $P$. aeruginosa 74 (17.96\%), Staphylococci sp. 98 $(23.79 \%)$ and Candida sp. 14 (3.4\%). Microbial isolates are shown in table 1.

Table 1: Distribution of isolated pathogens among total 412 clinical specimens.

\begin{tabular}{|l|c|c|}
\hline \multicolumn{1}{|c|}{ Type } & $\begin{array}{c}\text { No. of } \\
\text { isolates }\end{array}$ & Percentage \\
\hline P.aeruginosa & 74 & $17.96 \%$ \\
\hline $\begin{array}{l}\text { Enterobacteriaceae } \\
\text { sp. }\end{array}$ & 226 & $54.85 \%$ \\
\hline Staphylococci sp. & 98 & $23.79 \%$ \\
\hline Candida sp. & 14 & $3.4 \%$ \\
\hline Total & 412 & $100 \%$ \\
\hline
\end{tabular}

Distribution of $P$. aeruginosa isolates among different wards/ICUs. $P$. aeruginosa isolates were mostly isolated from Urology department representing 32.4\% (24/74) from total $P$. aeruginosa isolates, followed by General ICU representing 24.3\% (18/74), Chest ICU and neurology ICU representing $10.8 \%$ (8/74), internal medicine ICU 9.5\% (7/74), pediatric ICU $6.8 \%$ (5/74), and then postoperative ICU representing 5.4\% (4/74) are shown in figure 1 .

Antimicrobial susceptibility. The highest sensitivity was to imipenem 38 isolates $(51.3 \%)$. The antibiotic sensitivity then decrease in descending manner to be meropenem $(48.6 \%)>$ levofloxacin $(28.4 \%)>$ ciprofloxacin and ceftazidime $(21.6 \%)$ while the highest resistance rates were to carbenicillin 70 isolates $(94.6 \%)$ then gentamicin $(70.3 \%)$. The rate of resistance to antibiotics is shown in the table (2). Out of 74 $P$. aeruginosa isolates, 51 (69\%) strains were MDR and 23 (32\%) strains were non-MDR strains.

Determination for $\boldsymbol{P}$. aeruginosa isolates. All $P$. aeruginosa isolates (74 isolates) were tested for accurate determination of their MIC using the Imipenem (IP)-E test and Meropenem (MP)-E test strips. Results were interpreted using the breakpoints of CLSI (2016) as shown in figure 2.

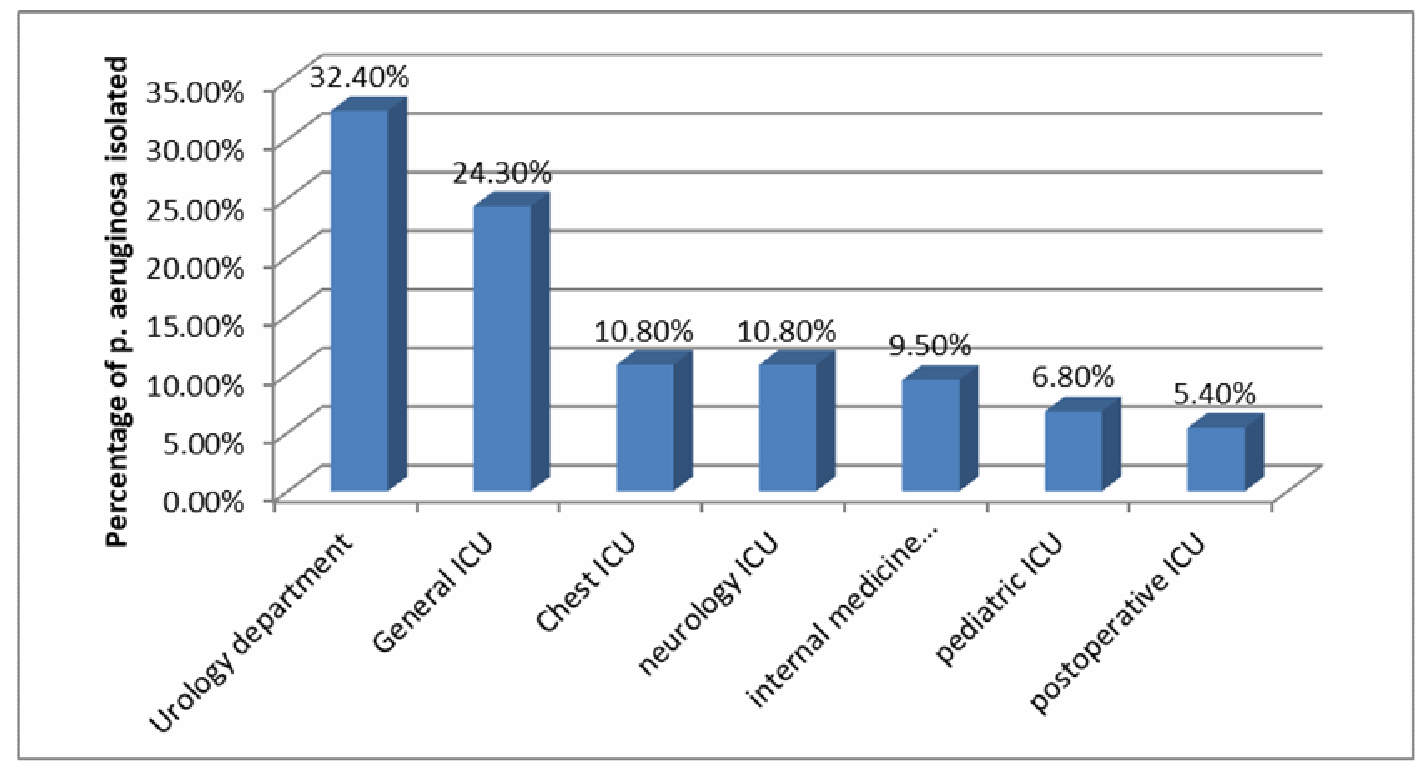

Fig. 1: Distribution of $P$. aeruginosa isolates among different wards/ICUs. 
Table 2: Antimicrobial susceptibility pattern of 74 P. aeruginosa urine isolates.

\begin{tabular}{|l|c|c|c|}
\hline \multicolumn{1}{|c|}{$\begin{array}{c}\text { Antimicrobial } \\
\text { drug }\end{array}$} & $\begin{array}{c}\text { No. of } \\
\text { resistant } \\
\text { isolates (\%) }\end{array}$ & $\begin{array}{c}\text { No. of } \\
\text { intermediate } \\
\text { isolates (\%) }\end{array}$ & $\begin{array}{c}\text { No. of } \\
\text { sensitive } \\
\text { isolates (\%) }\end{array}$ \\
\hline Imipenem & $27(36.5 \%)$ & $9(12.2 \%)$ & $38(51.3 \%)$ \\
\hline Meropenem & $29(39.2 \%)$ & $9(12.2 \%)$ & $36(48.6 \%)$ \\
\hline Ceftazidime & $49(66.2 \%)$ & $9(12.2 \%)$ & $16(21.6 \%)$ \\
\hline Amikacin & $43(58.1 \%)$ & $19(25.7 \%)$ & $12(16.2 \%)$ \\
\hline Gentamicin & $52(70.3 \%)$ & $9(12.2 \%)$ & $13(17.5 \%)$ \\
\hline Ciprofloxacin & $51(68.9 \%)$ & $7(9.5 \%)$ & $16(21.6 \%)$ \\
\hline Levofloxacin & $46(62.1 \%)$ & $7(9.5 \%)$ & $21(28.4 \%)$ \\
\hline Carbenicillin & $70(94.6 \%)$ & $1(1.4 \%)$ & $3(4 \%)$ \\
\hline
\end{tabular}

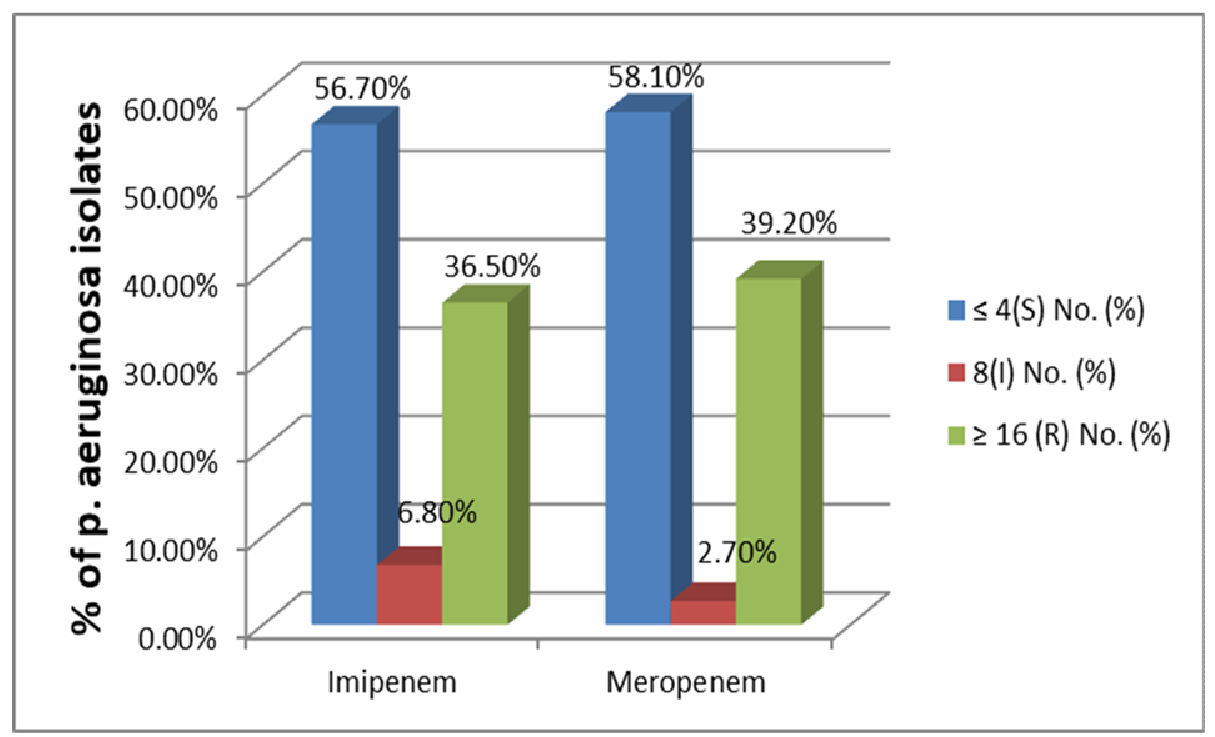

Fig. 2: Results of MIC of Imipenem and Meropenem using (IP)-E test and (MP)-E test strips in 74 P. aeruginosa isolates.

Detection of MBL by CD. Out of the $74 P$. aeruginosa isolates, $26(35.1 \%)$ and 25 isolates $(33.8 \%)$ showed an increase in the zone of inhibition of about $7 \mathrm{~mm}$ or more around IPMEDTA and MEM-EDTA disks compared to IPM and MEM disk alone (positive for M $\beta$ Ls production), respectively are shown in the figure 3 and table 3 .

Detection of MBL by DDST. Out of the total $74 P$. aeruginosa isolates, $23(31.1 \%)$ and 21 isolates (28.4\%) showed positive results for M $\beta$ Ls production using IPM and MEM, respectively, are shown in the figure 4 and table 4. but all IP and MP E-test susceptible $P$. aeruginosa isolates showed negative results of DDST.

\section{Discussion}

This study included patients suffering from nosocomial UTIs over a period of 17 months from November 2015 to April 2017. The aerobic pathogens causing UTI were identified. The antibiotic resistance pattern, for $P$. aeruginosa isolates was performed, the frequency of multidrug resistance among nosocomial $P$. aeruginosa infections, and carbapenems antibiotic sensitivity pattern of $P$. aeruginosa isolates were determined. Phenotypic detection of Metallo- $\beta$-lactamase production. 
Table 3: Results of CD test of all 74 P. aeruginosa isolates using IPM (10 $\mu \mathrm{g})$ and MEM $(10 \mu \mathrm{g})$ for $\mathrm{M} \beta \mathrm{L}$ detection.

\begin{tabular}{||c|c|c|c|c||}
\hline \multirow{2}{*}{ Sample } & \multicolumn{2}{|c|}{ CDT using IPM } & \multicolumn{2}{c|}{ CDT using MPM } \\
\cline { 2 - 5 } & Positive & Negative & Positive & Negative \\
\cline { 2 - 5 } & No. (\%) & No. $(\%)$ & No. $(\%)$ & No. (\%) \\
\hline $\begin{array}{l}\text { Total No of } \text { P. aeruginosa } \\
\text { isolates }(n=74)\end{array}$ & $26(35.1 \%)$ & $\begin{array}{c}48 \\
(64.9 \%)\end{array}$ & $\begin{array}{c}25 \\
(33.8 \%)\end{array}$ & $\begin{array}{c}49 \\
(66.2 \%)\end{array}$ \\
\hline
\end{tabular}

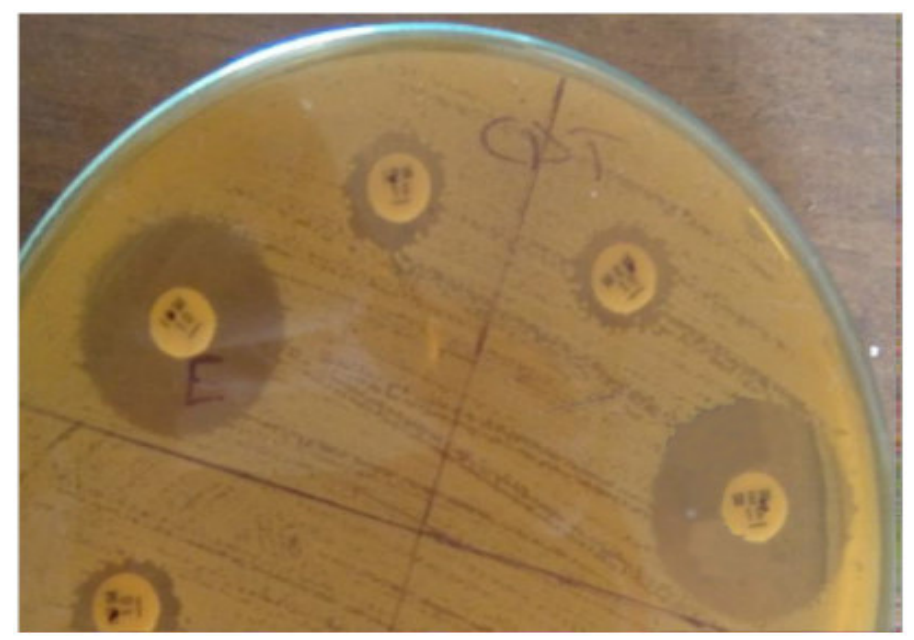

Fig. 3: Detection of $\mathrm{M} \beta \mathrm{L}$ in carbapenem resistant $P$. aeruginosa isolate by $\mathrm{CD}$ test $(+\mathrm{Ve})$.

Table 4: P. aeruginosa isolates using IPM $(10 \mu \mathrm{g})$ and MEM $(10 \mu \mathrm{g})$ for $\mathrm{M} \beta \mathrm{L}$ detection.

\begin{tabular}{||c|c|c|c|c|}
\hline \multirow{2}{*}{ Sample } & \multicolumn{2}{|c|}{ DDST using IPM } & \multicolumn{2}{c|}{ DDST using MPM } \\
\cline { 2 - 5 } & Positive & Negative & Positive & Negative \\
\cline { 2 - 5 } & No. $(\%)$ & No. $(\%)$ & No. $(\%)$ & No. $(\%)$ \\
\hline $\begin{array}{l}\text { Total No of } \text { P. aeruginosa } \\
\text { isolates }(n=74)\end{array}$ & $23(31.1 \%)$ & $51(68.1 \%)$ & $21(28.4 \%)$ & $53(71.6 \%)$ \\
\hline
\end{tabular}

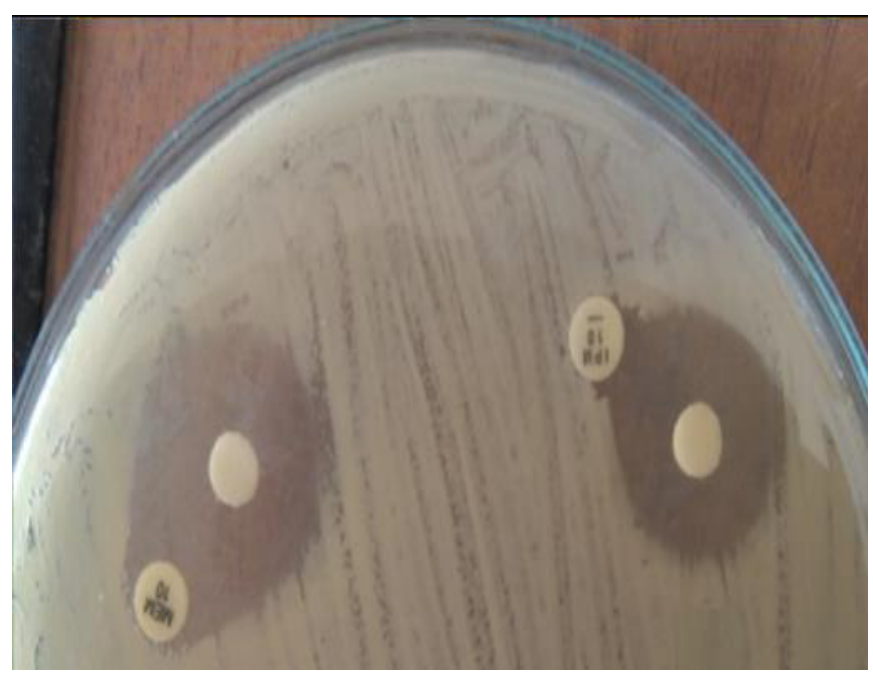

Fig. 4: Detection of M $\beta \mathrm{L}$ in carbapenem resistant $P$. aeruginosa isolate by DDST (+Ve). 
During the study period, rate of isolation of nosocomial $P$. aeruginosa urine isolates was $17.96 \%$ of isolated uropathogens. This finding agreed to some extent with that reported by Gad et al. ${ }^{12}$ who identified $P$. aeruginosa isolates in $18.2 \%$ of the clinical samples and Wassef $e t$ al. ${ }^{13}$ who identified $P$. aeruginosa isolates in $20.7 \%$ of different clinical samples, This rate were much higher than that reported by Aminizadeh and $\mathrm{Kashi}^{14}$, where the rate of incidence of $P$. aeruginosa in UTI was $13.2 \%$ In Malaysia, also Abbas et al. ${ }^{15}$ who identified $P$. aeruginosa isolates in $(12.5 \%)$ of the clinical samples and Mohammedi et al. $\frac{16}{}$ who identified $P$. aeruginosa isolates in $(12.35 \%)$ of different clinical samples. However, these results were less than those found by Mansour et al. ${ }^{17}$, who reported that the rate of isolation of Ps. aeruginosa from patient samples In Egypt and Saudi Arabia, was 32.8\% and 30.0\% respectively.

The leading uropathogens in this study were Enterobacteriaceae sp. (54.85\%). This was comparable to that reported by in Sudan reported by Shareef and Yagoub ${ }^{18}(68.01 \%)$ and to Milan and Ivan ${ }^{19}$ in Serbia (64\%). There were much higher rates reported in Nepal and Pakistan by Chandrashekhar et al. ${ }^{20}$ and Khan et $a l^{21}$. On the other hand rates ranging from (25) to $(37.4 \%)$ were reported in Japan and Turkey by Shigemura et al. ${ }^{22}$ and Savas et al. ${ }^{23}$

In this study Candida sp. forms (3.4\%) of isolated pathogens. This rate was more or less similar to $(3.7 \%)$ found in India by Hasan et $a l^{24}$, lower than reported in Kuwait $(16.5 \%)$ by Al Benwan et al. ${ }^{25}$. In Saudi Arabia, the frequency of nosocomial uro-pathogens isolates was studied by Alzohairy and Khadri ${ }^{26}$, where they showed that E. coli was the predominant pathogen and Klebsiella spp. were the second common organism in hospitalacquired infection followed by $P$. aeruginosa strains.

In our study, the highest number of $P$. aeruginosa strains were recovered from Urology department followed by General ICU then chest ICU and neurology ICU with percentages of $32.4 \%$ (24/74), $24.3 \%$ (18/74), $10.8 \%(8 / 74)$ and $10.8 \%(8 / 74)$, respectively (Fig. 1). These findings disagree to some extent with those of Javanbakht et al. ${ }^{27}$ who reported that Postoperative ICU were the most frequent category of infection $(46.5 \%)$ in Imam Reza hospital, followed by Urology department (22.7\%), and General ICU (9\%). These differences may be due to difference in the number of patients, place of study and genetic susceptibility.

The present study revealed that the resistance rates of $P$. aeruginosa were ranging from very high $(94.6 \%)$ to carbenicillin to moderate to ceftazidime $(66.2 \%)$ and high incidence of resistance can explain by our routine use of the drug (Table 2). These high values of resistance which were observed were comparable to those of the reports from Gujarat, with a resistance value of $(75 \%)^{28}$. Also, our results were consistent with data of Fazlul et $a l^{29}$, who stated that nosocomial $P$. aeruginosa urine isolates were highly resistant to ceftazidime $(61.9 \%)$.

In our study, the rate of resistance of $P$. aeruginosa urine isolates to imipenem was (36.5\%) and to meropenem was (39.2\%) in the same table. Our results were identical with those reported by Ashour and El-Sharif ${ }^{30}$ in Egypt who concluded that Acinetobacter and Pseudomonas species exhibited the highest resistance levels to imipenem (37.03\%) among other Gram-negative organisms.

Our results were higher than those reported by Wang et al. ${ }^{31}$ documented low rate of imipenem and meropenem-resistance among $P$. aeruginosa, it was (13\%) and (16\%) respectively. Queenan et al. ${ }^{32}$ stated that rate of imipenem and meropenem resistance among $P$. aeruginosa isolated from patients with UTIs was (18\%) and (22\%), respectively. Resistance rates to carbapenems may vary and depends on local antibiotic policies, origin of the strains, and geographic location ${ }^{33}$.

Our study showed marked increase in imipenem resistance $(39.2 \%)$ which may be attributed to increased use of imipenem in the hospital. This rate of carbapenem resistance reflects a threat limiting the treatment options in our hospitals. This can be explained in part by the increase in consumption of antimicrobial agents in the last decade leading to a selective pressure of antibiotics on $P$. aeruginosa and consequently the bacteria modify the resistant mechanisms. A similar high rate of resistance 
has been reported in many developing countries worldwide ${ }^{34}$.

The resistance rate of $P$. aeruginosa to gentamicin was $(70.3 \%)$ and amikacin was $(58.1 \%)$. In agreement with our result, Fazlul et $a .^{29}$, observed that the resistance of nosocomial $P$. aeruginosa urine isolates to gentamicin and amikacin was (75\%) and (48\%) respectively. Ramirez and Tolmasky ${ }^{35}$ reported much high rate of resistance to gentamicin (81\%). Sibi et al. ${ }^{36}$ found that resistance of $P$. aeruginosa to gentamicin and amikacin was (79\%) and (51\%), respectively. Zhao et al. ${ }^{37}$ found that the resistance rate of $P$. aeruginosa to gentamicin and amikacin was (77\%) and (61\%), respectively.

Fluoroquinolones are most commonly used in urinary tract infections. But resistance to fluoroquinolones is increasing. The resistance rate to ciprofloxacin was $(68.9 \%)$ and levofloxacin was (62.2\%). These results were similar to those reported by Gad et al. ${ }^{12}$, who found that most of the $P$. aeruginosa isolates were resistant to both levofloxacin (64\%), and ciprofloxacin (62\%). Abbas et al. ${ }^{15}$ also found that most of the $P$. aeruginosa isolates were resistant to levofloxacin (69.4\%), and ciprofloxacin (67.1\%). These results disagreed with the study of Kobayashi et al. ${ }^{38}$ also found that $(22.4 \%)$ of the P. aeruginosa isolates were resistant to ciprofloxacin. These rates were lower than that reported by Manjunath et $a l .{ }^{39}$ in India where the rate of ciprofloxacin resistance was (85\%).

In our study, the prevalence of MDR strains among $P$. aeruginosa urine isolates was 51 (69\%). Our result was coincides with studies that were done by Mohanasoundaram ${ }^{40}$ who found that, the percentage of the MDR in the $P$. aeruginosa strains had increased from (64\%) in 2008 to (71\%) in 2010; Alzohairy and Khadri $^{26}$ found that, P. aeruginosa $(58.3 \%)$ was the most common MDR uro-pathogens followed by E. faecalis (55.5\%) and E.coli (53.6\%). Our results was higher than Kirikae et $a l .{ }^{41}$ who reported that the percentage of MDR$P$. aeruginosa isolates in the urinary tract was significantly greater in both medical facilities and clinical laboratories, where the percentage of MDR strains was (43.8\%) and (41.6\%), respectively. However, the result was lower than $(76 \%)$ reported by El-Shouny et al. ${ }^{42}$.

In this study, phenotypic detection of carbapenemase producing $P$. aeruginosa isolates was done by determination of MICs of carbepenems using E-test. IP \& MP E-test identified 27 carbapenemase-producing $P$. aeruginosa isolates. IP E-test results showed that $36.5 \%$ (27/74) of $P$. aeruginosa isolates were imipenem resistant (MIC above 16 $\mu \mathrm{g} / \mathrm{ml})$, while $6.8 \%(5 / 74)$ of the isolates showed imipenem intermediate susceptibility (MIC $8 \mu \mathrm{g} / \mathrm{ml}$ ) and $56.7 \%(42 / 74)$ of the isolates were imipenem sensitive (MIC below 4 $\mu \mathrm{g} / \mathrm{ml}$ ) (Fig. 2). These results were similar to those reported by Arora et $a l^{43}$ who found that rate of imipenem- resistant among $P$. aeruginosa urine isolates was (37\%). In the Middle East the occurrence of imipenem resistant $P$. aeruginosa is alarmingly recognized. In Saudi Arabia, the resistance rate of $P$. aeruginosa to imipenem was increased to $(38.57 \%)$ in $2011^{44}$.

MP E-test results showed that $39.2 \%$ (29/74) of $P$. aeruginosa isolates were meropenem resistant (MIC above $16 \mu \mathrm{g} / \mathrm{ml}$ ), while $2.7 \%(2 / 74)$ of the isolates showed meropenem intermediate susceptibility (MIC 8 $\mu \mathrm{g} / \mathrm{ml})$ and $58.1 \%(43 / 74)$ of the isolates were meropenem sensitive (MIC below $4 \mu \mathrm{g} / \mathrm{ml}$ ). In agreement with our results, a study in Egypt reported a resistance rate of $(37.7 \%)$ to meropenem among $P$. aeruginosa isolated from hospitalized cancer patients ${ }^{45}$. This is explained by the differences in the pattern of antibiotic prescription and usage between the two studies.

In our study, CDT and DDST were done as phenotypic methods for detection of $\mathrm{M} \beta \mathrm{L}$ producing $P$. aeruginosa isolates including both carbapenem susceptible and nonsusceptible strains. By CDT, 35.1\% (26/74) and $33.8 \%(25 / 74)$ of $P$. aeruginosa isolates were $\mathrm{M} \beta \mathrm{L}$ producers phenotypically by IPM/EDTA and MEM/EDTA combined disks, respectively (Table 3 ). These findings are similar to high extent to the prevalence of MBL producers in Egyptian study which was (32.3\%) by Mansour et al. ${ }^{17}$, from UK (38.3\%) by Ellington et $a .^{46}$.

Regarding the DDST, $31.1 \%$ (23/74) and $28.4 \%(21 / 74)$ of $P$. aeruginosa isolates were 
$\mathrm{M} \beta \mathrm{L}$ producers phenotypically by IPM and MEM, respectively (Table 4). These results are in accordance with those of other studies in Egypt by Mansour et al. ${ }^{17}$ who reported that the prevalence of $\mathrm{M} \beta \mathrm{L}$ producers in Egyptian study was (32.3\%). In 2012, Polotto et al. ${ }^{47}$ surveyed $56 \mathrm{P}$. aeruginosa isolates by disk diffusion method and showed that 54/56 $(96.4 \%)$ of isolates were resistant to imipenem. They also showed that $17 / 56(30.3 \%)$ of imipenem-resistant strains were positive for production of MBL.

\section{Conclusion}

This study shows that there is increasing prevalence of $\beta$-lactamase producing $P$. aeruginosa in nosocomial infections in ALAzher University Hospital This is a major cause of concern as this implies that more and more instances of multidrug resistance are emerging. This leads to an overall negative impact on the health concerns and amounts to increasing difficulty in combating disease. It is recommended that awareness about antibiotic use and abuse be made a priority and measures of curbing unchecked use of prescription antibiotics be put into place. Establishment of infection control programs will help to lower the incidence of resistance in $P$. aeruginosa.

\section{REFERENCES}

1- A. Y. Peleg and D. C. Hooper, "Hospitalacquired infections due to gram-negative bacteria", New Engl. J. Med., 362 (19), 1804-1813 (2010).

2- J. A. Adachi, C. Perego, L. Graviss, T. Dvorak, R. Hachem, R.F. Chemaly, et al., "The role of interventional molecular epidemiology in controlling clonal clusters of multidrug resistant Pseudomonas aeruginosa in critically ill cancer patients", Am. J. Infect. Control., 37 (6), 442-446 (2009).

3- S. Sako, R. Kariyama, R. Mitsuhata, M. Yamamoto, K. Wada, A. Ishii, et al., "Molecular epidemiology and clinical implications of metallo- $\beta$-lactamaseproducing Pseudomonas aeruginosa isolated from urine", Acta. Med. Okayama, 68, 89-99 (2014).
4- S. Bratu, J. Quale, S. Cebular, R. Heddurshetti and D. Land-man, "Multidrug resistant Pseudomonas aeruginosa in Brooklyn, New York: molecular epidemiology and in-vitro activity of polymyxin B", Eur. J. Clin. Microbiol. Infect. Dis., 24, 196-201 (2005).

5- A. P. Magiorakos, A. Srinivasan, R. B. Carey, Y. Carmeli, M. E. Falagas, et al., "Multidrug-resistant, extensively drugresistant and pandrug-resistant bacteria: an international expert proposal for interim standard definitions for acquired resistance", Clin. Microbiol. Infect., 18, 268-281 (2012).

6- D. M. Livermore, "Multiple mechanisms of antimicrobial resistance in Pseudomonas aeruginosa: Our worst nightmare?", Clin. Infect. Dis., 34, 634640 (2002).

7- M. R. Franco, H. H. Caiaffa-Filho, M. N. Burattini and F. Rossi, "Metallobetalactamases among imipenem-resistant Pseudomonas aeruginosa in a Brazilian University Hospital", Clinics (Sao Paulo), 65 (9), 825-829 (2010).

8- R. E. Mendes, M. Castanheira, M. A. Toleman, H. S. Sader, R. N. Jones, and T. R. Walsh, "Characterization of an integron carrying bla IMP-1 and a new aminoglycoside resistance gene $\left(\operatorname{aac}\left(6^{\prime}\right)-31\right)$, and its dissemination among genetically unrelated clinical isolates in a Brazilian hospital", Antimicrob. Agents Chemother., 51, 2611-2614 (2007).

9- Clinical and Laboratory Standards Institute (CLSI). "Performance Standards for Antimicrobials Susceptibility Testing", Twenty First informational Supplement, Wayne, PA 2011, CLSI document, M100S21.

10- Clinical and Laboratory Standards Institute. "Performance Standards for Antimicrobial Susceptibility Testing", $26^{\text {th }}$ Edn., Wayne, PA 2016, CLSI document, M100-S21.

11- D. Yong, K. Lee, J. H. Yum, J. H. Shin, G. M. Rossolini and Y. Chong, "Imipenem-EDTA disk method for differentiation of MsL producing clinical 
isolates of Pseudomonas spp. and Acinetobacter spp.", J. Clin. Microbiol., 40, 3798-3801 (2002).

12- G. F. Gad, R. A. El-Domany, S. Zaki and H. M. Ashour, "Characterization of Pseudomonas aeruginosa isolated from clinical and environmental samples in Minia, Egypt: prevalence, antibiogram and resistance mechanisms", Journal of Antimicrobial Chemotherapy, 60, 10101017 (2007).

13- M. Wassef, H. El Mahallawy, M. M. Zafer, G. Doaa and R. Abdel hamid, "Lab Based Surveillance of Multidrug Resistant Pseudomonas aeruginosa in Cairo University Hospitals, Egypt", Journal of Microbiology \& Experimentation, 2 (2), 1-5 (2015).

14- Z. Aminizadeh and M. S. Kashi, "Prevalence of MDR and PDR among multiple Gram-negative spp.; experience in one teaching hospital, Tehran. Iran", International Research Journal of Microbiology, 2 (3), 090-095 (2011).

15- S. H. Abbas, M. Naeem, M. Adil, S. M. Naz, A. Khan and M. U. Khan, "Sensitivity patterns of Pseudomonas aeruginosa isolates obtained from clinical specimens in Peshawar", Journal of Ayub Medical College Abbottabad, 27, 329-332 (2015).

16- M. Mohammadi, E. Ghasemi, H. Mokhayeri, Y. Pournia and H. Boroun, "Antimicrobial resistance patterns of $E$. coli detected from hospitalized urine culture samples", Asian J. Biol. Sci., 3, 195-201 (2010).

17- S. Mansour, O. Eldaly, A. Jiman Fatani, M. Mohamed and E. Ibrahim, "Epidemiological characterization of Ps. aeruginosa isolates of intensive care units in Egypt and Saudi Arabia", East Mediter Health J., 19, 71-80 (2013).

18- S. A. Shareef and S. O. Yagoub, "Incidence and Isolation of Bacteria Associated With Nosocomial Urinary Tract Infection(UTI) in Sudanese Women", Research Journal of Microbiology, 5 (12), 1289-1294 (2010).

19- P. B. Milan and M. I. Ivan, "Catheterassociated and nosocomial urinary tract infections: antibiotic resistance and influence on commonly used antimicrobial therapy", Int. Urol. Nephro., 41, $461-464$ (2009).

20- R. N. Chandrashekhar, H. S. Joshi, M. Gurung, N. Shrestha and P. G. Shivananda, "Frequency and susceptibility profile of pathogens causing urinary tract infections at a tertiary care hospital in western Nepal", Singapore Med. J., 47 (4), 281-285 (2006).

21- B. A. Khan, S. Saeed, A. Akram, F. B. Khan and A. Nasim, "Nosocomial Uropathogens And Their Antibiotic Sensitivity Patterns In A Tertiary Referral Teaching Hospital In Rawalpindi, Pakistan", J. Ayub. Med. Coll. Abbottabad, 22 (1), 11-12 (2010).

22- K. Shigemura, K. Tanaka, H. Okada, Y. Nakano, S. Kinoshita, et al., "Pathogen occurrence and antimicrobial susceptibility of urinary tract infection cases during a 20-year period (1983-2002) at a single institution in Japan", Jap. J. Infect. Dis., 58, 303-308 (2005).

23- L. Savaş, N. Duran, N. Savaş, Y. Knlen and S. Ocak, "The prevalence and resistance patterns of Pseudomonas aeruginosa in intensive care units in a university hospital", Turk. J. Med. Sci., 35, 317-322 (2006).

24- A. S. Hasan, D. Nair, J. Kaur, G. Baweja, M. Deb and P. Aggarwal, "Resistance Patterns Of Urinary Isolates In A Tertiary Indian Hospital", J. Ayub. Med. Coll. Abbottabad, 19 (1), 39-41 (2007).

25- K. Al Benwan, N. Al Sweih and V. O. Rotimi, "Etiology and antibiotic susceptibility patterns of community- and hospital-acquired urinary tract infections in a General Hospital in Kuwait", Med. Princ. Pract., 19, 440-446 (2010).

26- M. Alzohairy and H. Khadri, "Frequency and antibiotic susceptibility pattern of uropathogens isolated from community and hospital-acquired infections in Saudi Arabia - A prospective case study", British Journal of Medicine \& Medical Research, 1 (2), 45-56 (2011).

27- A. Javanbakht, E. Askari, L. Danesh, N. Moghadas, I. Mostafavi and M. 
Naderinasab, "The incidence of cross infections in Imam Reza hospital, Mashhad, Iran", Iranian Journal of Microbiology, 4, 177-179 (2012).

28- W. H. Zhao and Z. Q. Hu ZQ. " $\beta$ lactamses identified in clinical isolates of Pseudomonas aeruginosa", Crit. Rev. Microbiol., 36, 245-258 (2010).

29- M. K. Fazlul, M. Z. Zaini, M. A. Rashid and M. H. Nazmul, "Antibiotic susceptibility profiles of clinical isolates of Pseudomonas aeruginosa from Selayang Hospital, Malaysia", Biomedical Research, 22 (3), 263-266 (2011).

30- H. M. Ashour and A. El-Sharif "Species distribution and antimicrobial susceptibility of gram-negative aerobic bacteria in hospitalized cancer patients", J. Transl. Med., 7 (1), 14 (2009).

31- J. Wang, J. Y. Zhou, T. T. Qu, P. Shen, et al., "Molecular epidemiology and mechanisms of carbapenem resistance in Pseudomonas aeruginosa isolates from Chinese Hospitals", Int. J. Antimicrob. Agents, 35, 486-491 (2010).

32- A.M. Queenan, W. Shang, K. Bush and R. K. Flamm, "Differential selection of single-step AmpC or efflux mutants of Pseudomonas aeruginosa by using cefepime, ceftazidime, or ceftobiprole", Antimicrob. Agents Chemother., 54, 4092-4097 (2010).

33- A. Singh, V. G. Richard, S. Shabbir, L.F. Steven and J. Z. Marcus, "Application of Molecular Techniques to the Study of Hospital Infection", Clin. Microbiol Rev., 19 (3), 512-530 (2006).

34- R. C. Picao, L. Poirel, A. C. Gales and P. Nordmann, "Diversity of $\beta$-lactamases produced by ceftazidime-resistant Pseudomonas aeruginosa isolates causing blood-stream infections in Brazil", Antimicrob. Agents Chemother., 53, 3908-3913 (2009).

35- M. S. Ramirez and M. E. Tolmasky, "Aminoglycoside modifying enzymes", Drug Resist., 13, 151-171 (2010).

36- G. Sibi, A. P. Devi, K. Fouzia and B. R. Patil, "Prevalence, microbiologic profile of UTI and its treatment with trimethoprim in diabetic patients", Res. $\boldsymbol{J}$. Microbiol., 6 (6), 543-551 (2011).

37- W. H. Zhao, G. Chen, R. Ito and Z. Q. Hu, "Relevance of resistance levels to carbapenems and integron-borne blaIMP1, blaIMP-7, blaIMP-10 and blaVIM-2 in clinical isolates of Pseudomonas aeruginosa", J. Med. Microbiol., 58, 1080-1085 (2009).

38- H. Kobayashi, M. Isozaki, T. Fukuda, Y. Anzai and F. Kato, "Surveillance of fluoroquinolone-resistant clinical isolates of Pseudomonas aeruginosa", Op. J. Med. Microbiol., 3, 144-150 (2013).

39- G. N. Manjunath, R. Prakash, V Annam and K. Shetty, "Changing trends in the spectrum of antimicrobial drug resistance pattern of uropathogens isolated from hospitals and community patients with urinary tract infections in Tumkur and Bangalore", Int. J. Biol. Med. Res., 2 (2), 504-507 (2011).

40- K. M. Mohanasoundaram, "The antimicrobial resistance pattern in the clinical isolates of Pseudomonas aeruginosa in a Tertiary Care Hospital., 2008-2010 (A 3 Year Study)", Journal of Clinical and Diagnostic Research, 5 (3), 491-494 (2011).

41- T. Kirikae, Y. Mizuguchi and Y. Arakawa, "Investigation of isolation rates of Pseudomonas aeruginosa with and without multidrug resistance in medical facilities and clinical laboratories in Japan", J. Antimicrob. Chemother., 61 (3), 612-615 (2008).

42- W. A. El-Shouny, S. S. Ali, J. Sun, S. M. Samy and A. Ali, "Drug resistance profile and molecular characterization of extended spectrum $\beta$-lactamase (ES $\beta \mathrm{L}$ )producing Pseudomonas aeruginosa isolated from burn wound infections. Essential oils and their potential for utilization", Microbial Pathogenesis, 116, 301-312 (2018).

43- D. Arora, N. Jindal, R. Kumar and A. Romit, "Emerging antibiotic resistance in Pseudomonas aeruginosa challenge", Int. J. Pharm. Pharm. Sci., 3 (2), 82-84 (2011). 
44- A. A. Mohamed, A. M. Shibl, S. A. Zaki and A. F. Tawfik, "Antimicrobial resistance pattern and prevalence of metallo- $\beta$-lactamases in Pseudomonas aeruginosa from Saudi Arabia", African Journal of Microbiology Research, 5 (30), 5528-5533 (2011)

45- J. W. Decousser, P. Pina, F. Picot, C. Delalande, et al., "Frequency of isolation and antimicrobial susceptibility of bacterial pathogens isolated from patients with bloodstream infections: a French prospective national survey", $\boldsymbol{J}$. Antimicrob. Chemother., 51 (5), 12131222 (2003).
46- M. J. Ellington, J. Kistler, D. M. Livermore and N. Woodford, "Multiplex PCR for rapid detection of genes encoding acquired metallo- $\beta$-lactamases", Antimicrob. Chemother., 59 (2), 321-322 (2007).

47- M. Polotto, T. Casella, M. G. Oliveira, F. G. Rubio, M. L. Nogueira, et al., "Detection of Pseudomonas aeruginosa harboring blaCTX-M-2, blaGES-1 and blaGES 5, blaIMP-1 and blaSPM-1 causing infections in Brazilian tertiarycare hospital", BMC. Infect. Dis., 12, 176 (2012). 


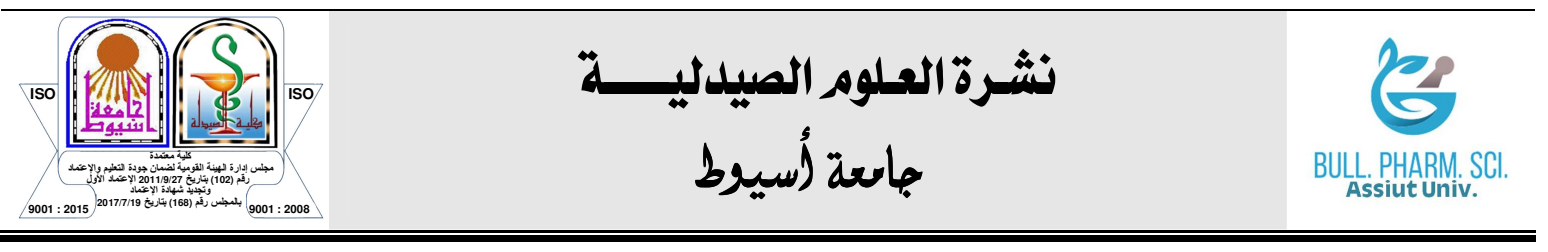

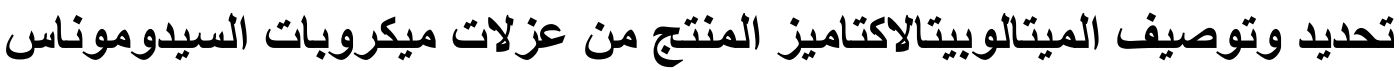

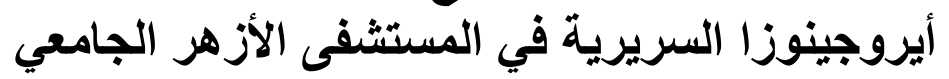

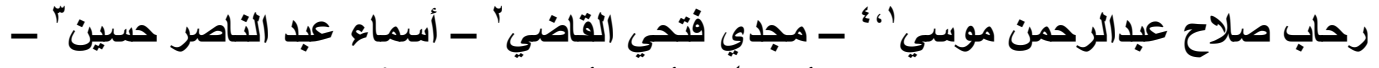

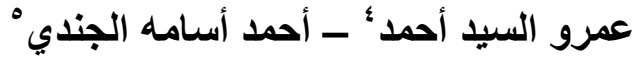

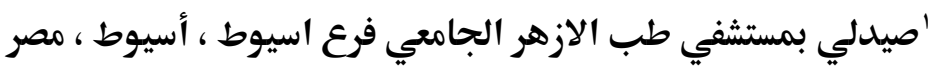

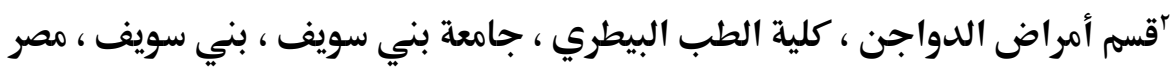

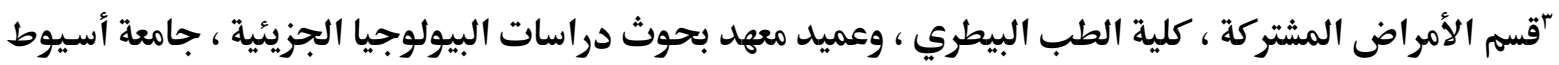

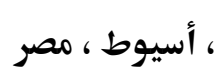

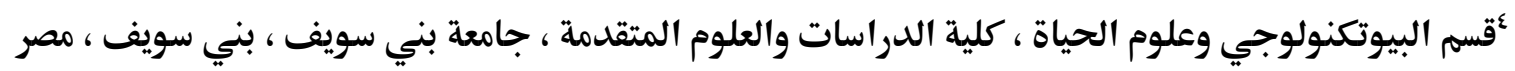

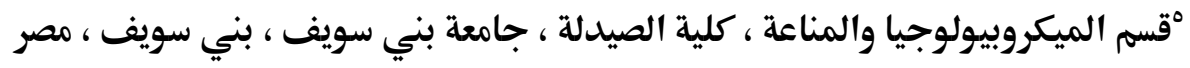

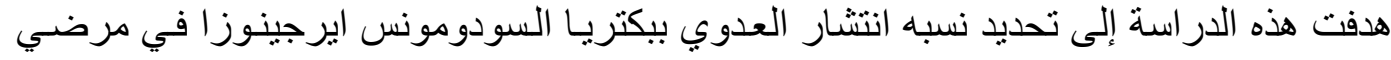

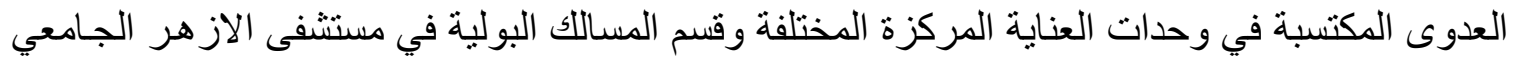

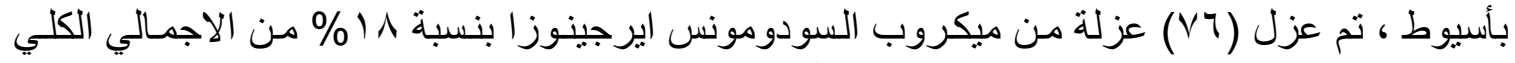

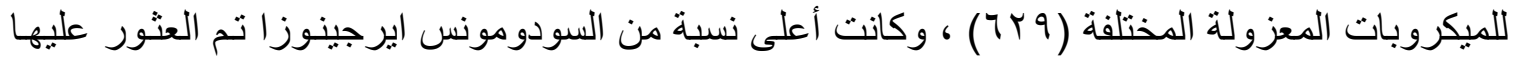

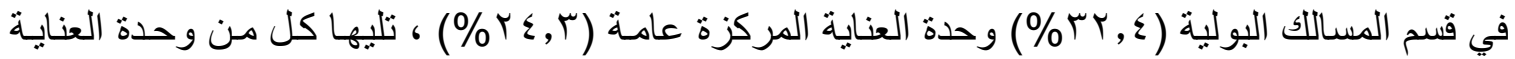

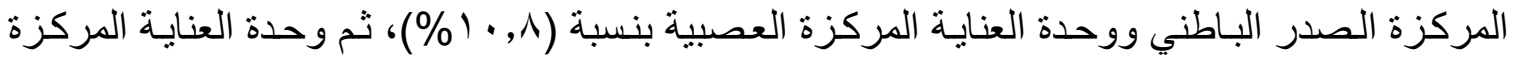

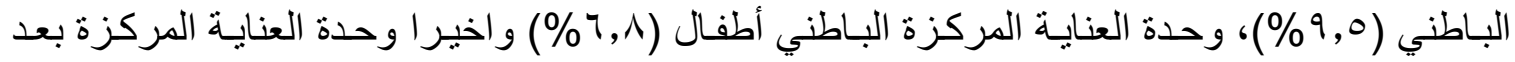

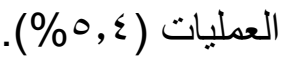

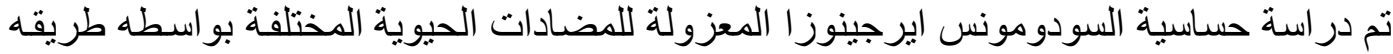

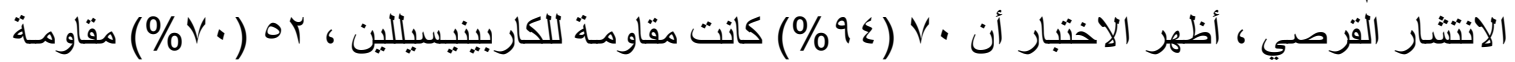

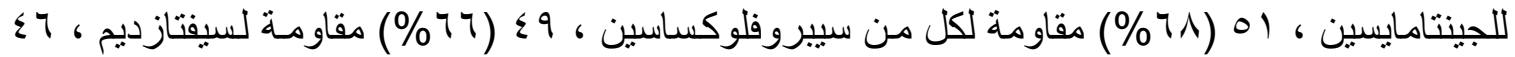

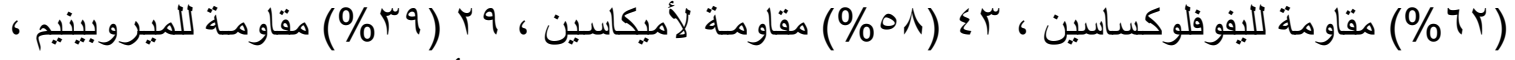

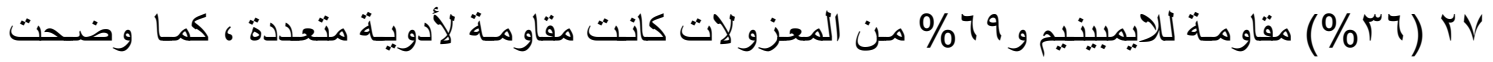

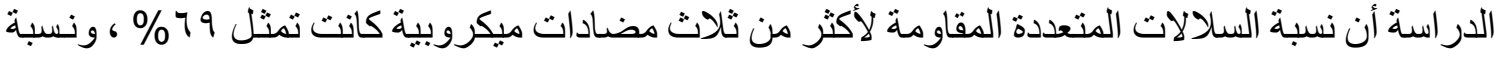

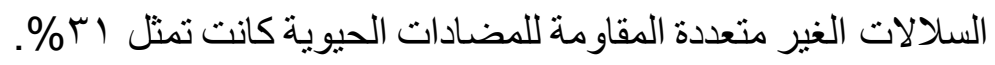

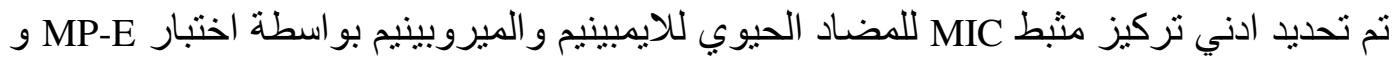

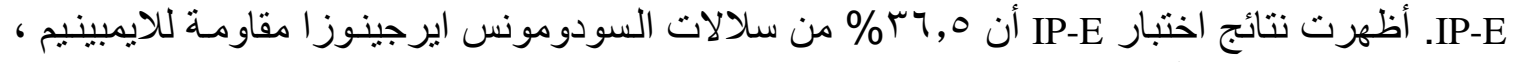

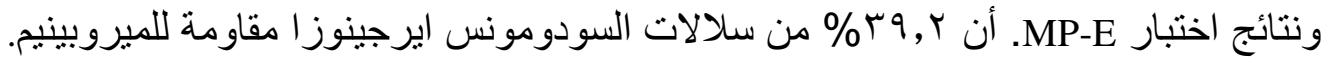

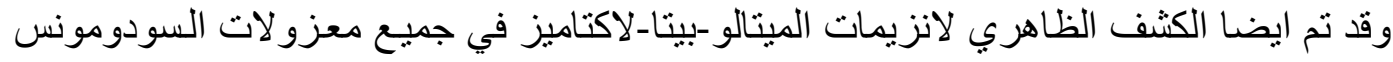

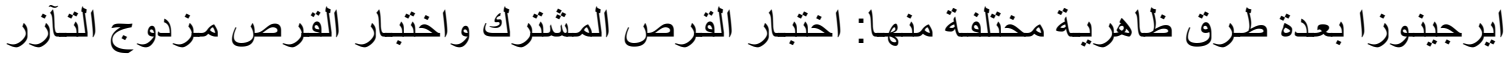

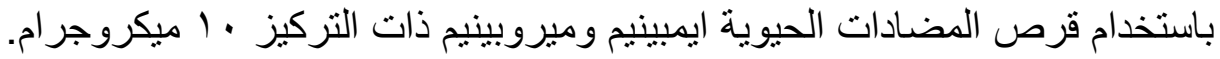

